\title{
THE EFFECT OF PLATELET RICH-FIBRIN WITH AND WITHOUT AUTOGENOUS BONE GRAFT ON BONE REGENERATION FOLLOWING ENUCLEATION OF MAXILLARY CYSTS
}

\author{
Alaa M. Zain Elabdin ${ }^{1 *} B D S$, Ahmed A. Sharara ${ }^{2} P h D$, Hala R. Ragab ${ }^{3} P h D$
}

\begin{abstract}
INTRODUCTION: Reconstruction of bony defects represents a challenging problem for the surgical community. Autologous bone grafting involves utilizing bone obtained from the same individual receiving the graft. Bone can be harvested from the mandibular symphysis (chin area). Autologous platelet rich-fibrin (PRF) is considered to be a healing biomaterial, which has been widely used for accelerating soft and hard tissue regeneration. The use of bone graft in combination with platelet rich-fibrin helps in rapid bone regeneration when compared to natural healing.

OBJECTIVES: is to compare the effect of platelet rich-fibrin with and without autogenous bone graft on bone regeneration following enucleation of maxillary cysts.

MATERIALS AND METHODS: Twenty-one patients with maxillary cysts had been treated with autogenous bone graft with platelet-rich fibrin versus platelet rich-fibrin without autogenous bone graft following cyst enucleation, and the follow up was done for 6 months using Cone Beam Computerized Tomography (CBCT) to evaluate bone regeneration in the defect site.

RESULTS: All groups showed increasing in bone density. Group A showed the best results with a mean 330.71, while Group B showed results with a mean 184 . Group C showed results with a mean 117.71 .

CONCLUSIONS: PRF can be considered a healing biomaterial, as it features all the necessary parameters permitting bone regeneration without any adverse reaction. The addition of autogenous bone graft to PRF accelerates the regenerative capacity of bone. They give a predictable clinical and radiographic evidence of bone formation and faster healing than using PRF alone.

KEYWORDS: maxillary cyst, autogenous bone graft, platelet rich fibrin
\end{abstract}

1. Bachelor student of Oral and Maxillofacial Surgery, Department of Oral and Maxillofacial Surgery, Faculty of Dentistry, Alexandria University, Egypt, Dentist at Ministry of Health, Alexandria Egypt.

2. Professor of Oral and Maxillofacial Surgery, Department of Oral and Maxillofacial Surgery, Faculty of Dentistry, Alexandria University, Egypt.

3. Assistant Professor of Oral and Maxillofacial Surgery, Department of Oral and Maxillofacial Surgery, Faculty of Dentistry, Alexandria University, Egypt.

* Corresponding author:

E-mail: alaa.zain.surgeon.az@gmail.com

\section{INTRODUCTION}

Reconstruction of bony defects represents a challenging problem for the surgical community. Spontaneous bone regeneration in untreated defects is limited to small areas because of the rapid proliferation of surrounding soft tissue (1).

Bone grafts and bone regeneration materials are being used with varying degrees of success. These approaches are known as regenerative therapies. The key to tissue regeneration is to simulate a cascade of healing events that, if coordinated, can result in the completion of integrated tissue formation (2).

Grafting materials include autografts, allografts, xenografts and synthetic bone substitutes. Many consider autologous bone grafts the gold standard, but their disadvantages are the limited availability, and the necessity for additional operation, and the risk of post-operative pain, parasthesia and infection. Alternatively, xenografts and allografts have been well documented to be suitable as bone replacement grafts (3-5). However, the incomplete resorption of these materials has frequently been reported moreover, a risk of transmitting diseases still exists from their use (6-8).

Platelet-rich fibrin was first introduced in France by Choukroun et al in 2006 (9). It was considered a secondgeneration platelet concentrate as it is produced without any anticoagulants or gelifying agents. PRF is being completely autogenous in nature, simple and relatively inexpensive with a specific three-dimensional architecture of the fibrin. PRF has also shown increased levels of inflammatory and healing cytokines. After activation of the platelets which are trapped within fibrin matrix, growth factors are released and stimulate the mitogenic response in the bone periosteum during normal wound healing for repair of the bone. Better understanding of physiologic properties of platelets in wound healing since the last two decades led to an increase in its therapeutic applications in the various forms showing varying results. PRF represents a new revolutionary step in the platelet gel therapeutic concept (10).

The aim of the work is to compare the effect of platelet rich-fibrin with and without autogenous bone graft on bone regeneration following enucleation of maxillary cysts.

\section{MATERIALS AND METHODS \\ Patients}

Appropriate ethical clearance was taken from the Oral and Maxillofacial Surgery Department, Faculty of Dentistry, Alexandria University where the study was carried out. Also, that an informed consent was signed by the patients participating in the study.

This study will be conducted on Twenty-one patients diagnosed with maxillary cysts selected from the Outpatient 
Clinic of the Oral and Maxillofacial Surgery Department, Faculty of Dentistry, Alexandria University.

After enucleation of the cysts, the Patients will be divided into three groups;

Group A: (7 patients) the defects will be filled with platelet rich-fibrin PRF and autogenous bone graft.

Group B: (7 patients) the defect will be filled with platelet rich-fibrin PRF without autogenous bone graft.

Group C: (7 Patients) the defect will be allowed to heal without any grafting material.

Inclusion criteria

- Patients diagnosed with maxillary cyst not more than 2x2cm in size.

- Patient's age ranges between 20-50 years.

\section{Exclusion criteria}

- Any systemic condition that may compromise bone healing such as: immunological diseases, uncontrolled diabetes mellitus, history of chemotherapy or radiotherapy, haematological disorder, patients under anticoagulant therapy.

- Presence of infection at the surgical site.

Materials

1-Autogenous bone graft

Autogenous bone will be harvested using bone harvester auto-max system from chin area. A drill with $0.7 \mathrm{~mm}$ diameter had been used with $4 \mathrm{~mm}$ depth stopper (Figure 1).

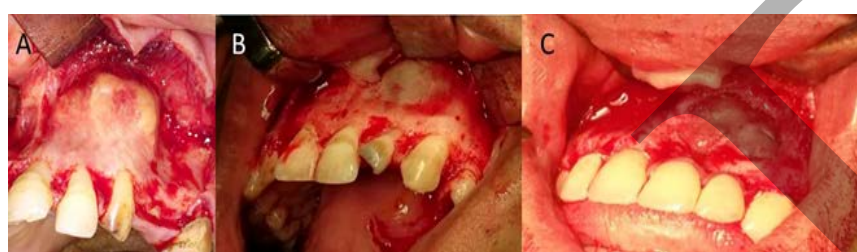

Figure (1): Reflection of mucoperiosteal flap.

\section{2-Platelet Rich- Fibrin}

Twenty milliliters of whole venous blood will be collected in 2 sterile $10 \mathrm{ml}$ glass test tubes without anticoagulant. Then the test tubes will be placed in a table centrifuge machine (Centrifuge machine to form fibrin) at 3000 revolutions per minute (RPM) for 10 minutes.

\section{Methods}

\section{I.Pre-surgical phase}

\section{1-History taking}

a) Chief complaint.

b)Duration, cause, site of the lesion.

c) History of previous intervention.

d)History of any type of medication taken by the patient.

\section{Clinical examination}

C. Inspection: for determining facial asymmetry, single or multiple neck masses, anatomical site of the lesion, size, shape, surface color and texture, as well as examination of oro-pharynx, tongue, floor of the mouth, palate and oral mucosa.

D. Palpation: lymph nodes and temporomandibular joint examination. Lesion examination for determining consistency, tenderness, fluctuation, definition of boundaries, pulsation, mobility of the skin or mucosa overlying the lesion.

E. Root canal treatment was performed for the teeth related to the maxillary cystic lesion.

F. Radiographic examination

Orthopantomogram (OPG): was performed to assess the site and relating to vital structures.
Cone Beam Computed Tomography (CBCT): was performed to assess the size, site, shape, extension and the relation to vital structures.

\section{II.Surgical phase}

1-The procedure was performed under complete aseptic conditions, and the surgical field was swabbed with povidone Iodine (Betadine ${ }^{\circledR}$, Nile Company for Pharm., Chemical IND., Cairo) solution before the operation.

2-The surgical procedure was performed under local or general anesthesia, depending on the extent of the lesion and the condition of the patient.

3-A full thickness pyramidal mucoperiosteal flap was performed using Bard-Parker blade no: 15 in the buccal aspect, extending one tooth mesially and distally to the cystic lesion to allow excellent inspection and management.

4-The mucoperiosteal flap was reflected using a periosteal elevator and bone was exposed (Figure 1).

5-The pathological lesion was exposed using a bone rongeur and rose head bur on low speed hand piece under copious irrigation by normal saline.

6-The whole pathological tissues were carefully enucleated and sent for histopathological examination (Figure 2).

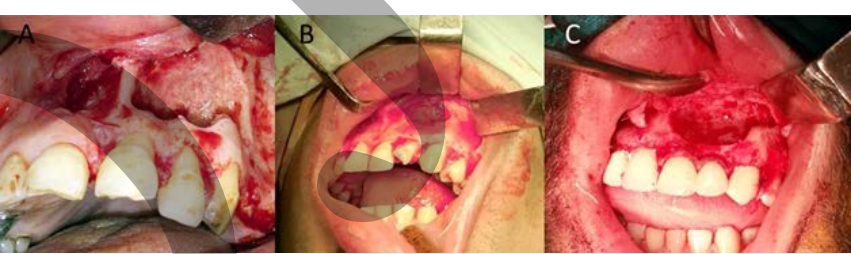

Figure (2): Enucleation of the pathological tissues.

7-Apicectomy was performed for the teeth that were involved in the lesion using a fissure bur on the high-speed hand piece and unsolvable teeth were extracted.

8-The cavity was curetted again to remove any remaining tissues and was irrigated with $0.9 \%$ normal saline.

9-20 $\mathrm{ml}$ of venous blood were collected from group A and group B patients and PRF was prepared.

10-The Full thickness mucoperiosteal flap was performed using Bard-Parker blade no: 15 in the chin area in group A patients to harvest autogenous bone graft.

11-In cases of group A, the bone defect was filled with PRF and auotgenous bone graft, while in cases of group $B$, the bone defect was filled with PRF. Group C patients were allowed to heal without graft material (Figure 3).

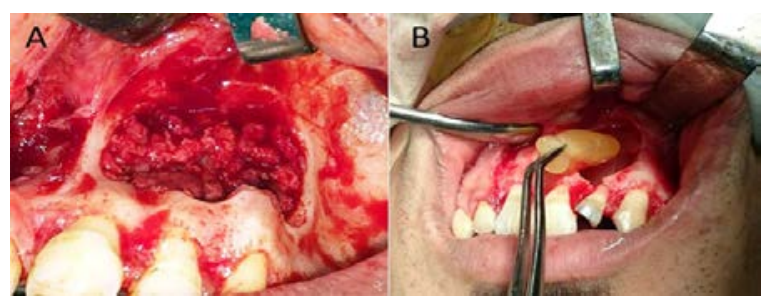

Figure (3): A) Grafting with autogenous bone graft with prf. B) Grafting only with prf.

12-The flap was then repositioned and sutured with interrupted 3-0 Vicryl sutures.

III. Postsurgical phase

Post-operative instructions were given to the patients including:

- Cold packs on the first day.

- Oral hygiene instructions. 
- Soft diet was recommended for one week post-operatively. Post-operative medications including:

- Antibiotic: Amoxicillin-Clavulanic acid (Hibiotic, Amoun pharmaceutical CO. S.A.E. - Egypt) (1 GM/12 hours) was prescribed for 5 days post-operatively.

- Analgesic: diclofenac potassium (Cataflam, Novartis, Switzerland) (50 mg/8 hours) prescribed for 3 days.

- Mouthwash: patients were instructed to use chlorohexidine mouth wash (Hexitol mouth wash: by Arab drug company, Cairo, Egypt.) twice daily for one week, starting on the 2nd day post-operative.

- The sutures were removed after seven days postoperatively.

IV. Follow up phase

A. Clinical evaluation

\section{A- Early post-operative follow up}

Post-operative clinical examination and follow-up were performed on the third and seventh day post-operatively then weekly for the first month for the following:

\section{1-Post-operative pain $\&$ discomfort}

Evaluation of pain \& discomfort was performed using visual analogue pain scale (VAS). The VAS is a simple, reproducible scale that allows the severity of the pain experienced to be expressed as a numeric value. The VAS is represented as a plain horizontal $10-\mathrm{cm}$ line. The patients were instructed to bisect the line at a point appropriate to their present discomfort. A zero-value regarded to be pain free, whereas the most severe pain was rated at 10. (It was arbitrarily defined a score of 9 or 10 as very severe, 7 or 8 as severe, 5 or 6 as moderate, 3 or 4 as mild, and 1 or 2 as asymptomatic) (11).

2-Post-operative healing

Healing was evaluated clinically regarding the presence of any bleeding, pus, signs of inflammation, any dehiscence in the wound margins, or any abnormal reaction to the bone graft.

\section{B- Delayed post-operative follow up}

Clinical evaluation: all the patients were re-examined post operatively at three months and six months intervals for any clinical complication.

B. Radiographic evaluation: all the patients had been examined with CBCT at 6 months postoperatively.

\section{Statistical analysis of the data}

Data was fed to:

\section{Student t-test.}

2. ANOVA with repeated measures.

\section{RESULTS}

This study was conducted on twenty-one patients diagnosed with maxillary cysts selected from the Outpatient Clinic of the Oral and Maxillofacial Surgery Department, Faculty of Dentistry, Alexandria University.

\section{Clinical Data (Table 1)}

- The patients were thirteen males (62\%) and eight females (38\%) aged 20 to 40 years with an average age of 29 years divided into three groups (Table1).

- Nine patients had undergone surgery with local anesthesia, while twelve patients had undergone surgery with general anesthesia. The type of anesthesia had been chosen depending on the relation of the cyst to vital structures and the condition of the patient.

- The results of the histopathologic examination showed that:
- In group A: six patients had radicular inflammatory cysts and one patient had dentigerous.

- In group B: six patients had radicular inflammatory cysts and one patient had multiple keratocysts (Gorlin-Golz syndrome).

- In group c: five patients had radicular cysts and two patients had two dentigerous cysts.

Table (1): Comparison between the three studied groups according to demographic data.

\begin{tabular}{|c|c|c|c|c|c|c|c|c|}
\hline & \multicolumn{2}{|c|}{$\begin{array}{c}\text { Group A } \\
(\mathbf{n}=7)\end{array}$} & \multicolumn{2}{|c|}{$\begin{array}{c}\text { Group B } \\
(\mathbf{n}=7)\end{array}$} & \multicolumn{2}{|c|}{$\begin{array}{c}\text { Group C } \\
(n=7)\end{array}$} & \multirow{2}{*}{$\begin{array}{c}\text { Test } \\
\text { of } \\
\text { Sig. }\end{array}$} & \multirow{2}{*}{$\mathbf{p}$} \\
\hline & No. & $\%$ & No. & $\%$ & No. & $\%$ & & \\
\hline \multicolumn{9}{|l|}{ Gender } \\
\hline Male & 5 & 71.4 & 3 & 42.9 & 5 & 71.4 & $\chi^{2}=$ & ${ }^{\mathrm{MC}} \mathrm{p}=$ \\
\hline Female & 2 & 28.6 & 4 & 57.1 & 2 & 28.6 & 1.578 & 0.622 \\
\hline \begin{tabular}{|l|} 
Age (years) \\
\end{tabular} & \multicolumn{2}{|l|}{ 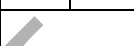 } & \multirow{2}{*}{\multicolumn{2}{|c|}{$22.0-40.0$}} & \multirow{2}{*}{\multicolumn{2}{|c|}{$20.0-35.0$}} & \multirow{4}{*}{$\begin{array}{c}\mathrm{F}= \\
1.671\end{array}$} & \multirow{4}{*}{0.216} \\
\hline & $\begin{array}{r}20 \\
3\end{array}$ & & & & & & & \\
\hline Mean \pm SE & & & \multicolumn{2}{|c|}{$30.29 \pm 7.50$} & \multicolumn{2}{|c|}{$28.0 \pm 4.55$} & & \\
\hline Median & & & \multicolumn{2}{|c|}{300} & \multicolumn{2}{|c|}{28.0} & & \\
\hline
\end{tabular}

$\chi^{2}$, p: $\chi^{2}$ and $p$ values for Chi square test

${ }_{\mathrm{MC}} \mathrm{p}$ : p value for Monte Carlo for Chi square test for comparing between the three groups

F,p: $F$ and p values for ANOVA test

\section{Post-operative evaluation}

I- Clinically

1. Post-operative pain, swelling and discomfort:

Clinically, all cases showed moderate edematous swelling 24 hours after the surgery, which gradually subsided over a period of five days. There was no unusual or severe pain, just mild pain at the site of operation which subsided totally on the third day post-operatively.

\section{Post-operative healing}

Healing was uneventful in all cases with no post-operative complications. There was absence of bleeding, pus, signs of inflammation or any dehiscence in the wound margins or extrusion of material during removal of the sutures on the seventh day post-operatively.

\section{II- Radiographically}

1. Measurement of bone density in bone defects by CT scan Group A

Immediate postoperative the mean density of the bone defects was $87.71 \mathrm{HU}$, after six months it was $418.43 \mathrm{HU}$. The increase in the bone density was significant after six months at $\mathrm{P}<0.001$.

\section{Group B}

Immediate postoperative the mean density of the bone defects was 29.29 HU, after six months it was 210.29 HU. It revealed that the increase in bone density was statistically significant between immediately postoperative and after six months at $\mathrm{P}<0.001$.

\section{Group C}

Immediate postoperative the mean density of the bone defects was $19.0 \mathrm{HU}$, after six months it was $136.71 \mathrm{HU}$. It revealed that the increase in bone density was statistically significant between immediately postoperative and after six months at $\mathrm{P}<0.001$.

Comparison between the bone Density of the three groups after 6 months using Hounsfield Unit

The mean change of the bone density was 330.71 HU for group A, while, the mean change of bone density was $\mathrm{HU}$ 
for group B. Finally, the mean change of bone density was 117.71 HU for group C.

It revealed that the increase in bone density in group $\mathrm{A}$ was statistically significant than in group $\mathrm{B}$ and $\mathrm{C}$ at $\mathrm{P}<$ 0.001 (Table 2).

Table (2): Comparison between the three studied groups according to changes of bone density (Houns Field).

\begin{tabular}{|c|c|c|c||}
\hline \multirow{2}{*}{ Patient No. } & \multicolumn{3}{|c|}{ Change (Immediately post-operative/ after 6 } \\
months)
\end{tabular}

F,p: $F$ and p values for ANOVA test, Sig. bet. grps was done using Post Hoc Test (LSD)

$\mathrm{p}_{1}$ : $\mathrm{p}$ value for comparing between group $\mathrm{A}$ and group $\mathrm{B}$ $\mathrm{p}_{2}$ : $\mathrm{p}$ value for comparing between group $A$ and group $C$ p3: $\mathrm{p}$ value for comparing between group $\mathrm{B}$ and group $\mathrm{C}$

$*$ : Statistically significant at $\mathrm{p} \leq 0.05$

\section{Measurement of surface area of bone defects by CT scan Group A}

Immediate postoperative the mean surface area of the bone defects was $22.75 \mathrm{~mm} 2$, after six months it was $6.14 \mathrm{~mm} 2$. The decrease in the surface area was significant after six months at $\mathrm{P}<0.001$.

\section{Group B}

Immediate postoperative the mean surface area of the bone defects was $20.71 \mathrm{~mm} 2$, after six months it was $10.14 \mathrm{~mm} 2$. The decrease in the surface area was significant after six months at $\mathrm{P}<0.001$.

\section{Group C}

Immediate postoperative the mean surface area of the bone defects was $25.29 \mathrm{~mm} 2$, after six months it was $14.29 \mathrm{~mm} 2$. The decrease in the surface area was significant after six months at $\mathrm{P}<0.001$.

Comparison between the surface area of the bone defect of the three groups after 6 months

The mean change in the bone defects surface area was 16.43 $\mathrm{mm} 2$ for group $\mathrm{A}$, while the mean change in the bone defects surface area was $10.57 \mathrm{~mm} 2$ for group B, while the mean change in the bone defects surface area was 11.0 for group C (Figures 4, 5, 6).

Decrease in the bone defect surface area was statistically significant in all groups. In comparison, the decrease in surface area among the three groups at 6 months post-operative it was statistically non-significant at $\mathrm{P}<$ 0.001 (Table 3).

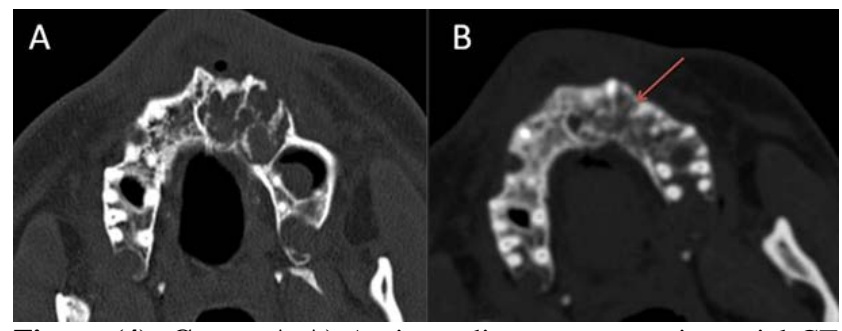

Figure (4): Group A A) An immediate post-operative axial CT showing bone defect following cyst enucleation. B) An axial CT 6 months post-operative (increase in bone density, bone regeneration, formation of buccal cortical plate).

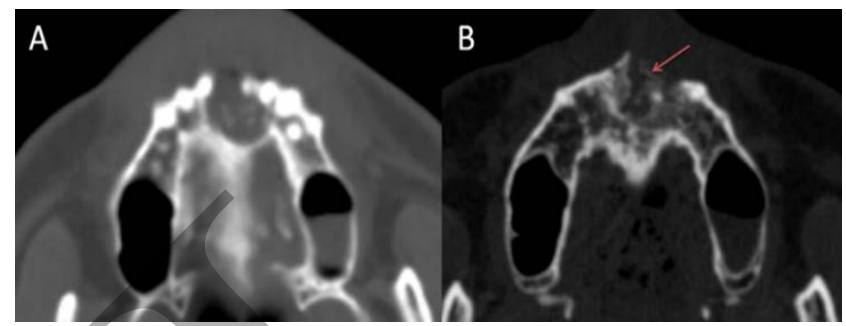

Figure (5): Group B A) An immediate post-operative axial CT showing bone defect following cyst enucleation. B) An axial CT 6 months post-operative showing (bone regeneration in the defect and formation of the cortical plate).

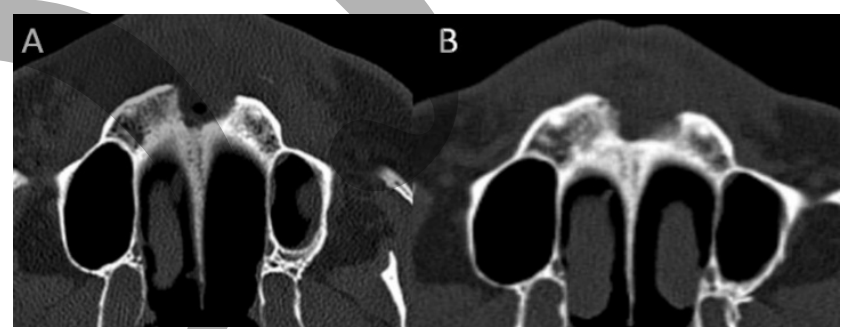

Figure (6): Group C A) An immediate post-operative axial CT showing bone defect following cyst enucleation. B) An axial CT 6 months post-operative showing (bone regeneration in the borders of the defect).

Table (3): Comparison between the three studied groups according to changes of Bone defect surface area (mm2)

\begin{tabular}{|c|c|c|c|}
\hline \multirow{2}{*}{ Patient No. } & \multicolumn{3}{|c|}{ Change (Immediately post-operative/ after 6 } \\
months)
\end{tabular}

F,p: $\mathrm{F}$ and $\mathrm{p}$ values for ANOVA test, Sig. bet. grps was done using Post Hoc Test (LSD)

*: Statistically significant at $\mathrm{p} \leq 0.05$

\section{DISCUSSION}

The diagnosis and treatment of bone cysts of the jaws, is very common in oral and maxillofacial surgery. The treatment objective is restoring the morphology and function of the affected area (12).

Bone regeneration using PRF was selected in this study as it is the latest development among the platelet concentrates. It is a second-generation platelet concentrate 
widely used to accelerate soft and hard tissue healing and is a strictly autologous fibrin matrix containing a large quantity of platelet and leukocyte cytokines (13).

Autogenous grafts are still considered to be the gold standard as they are the most predictable material for regeneration. They provide the added benefits of eliminating the risk of immunologic reaction or disease transmission. However, the limited amount of autogenous bone obtained by time-consuming methods as well as their susceptibility to microbial contamination is the major drawback during procurement from intraoral sites (14).

Carini et al in 2014 (15) did a systematic review autologous bone versus autologous bone and bone substitutes. A systematic review of the major online databases (MEDLINE, PUBMED and SCIENCE DIRECT) and a manual search of the most relevant articles from journals with Impact Factor was carried out covering the period between 1974 and 2013. Several dental journals were searched manually, in particular those specialized in periodontal and oral- maxillofacial surgery. They focused their review on articles that compared GBR technique with several kinds of bone: autogenous bone (100\%); a mixture of autogenous and heterologous bone (50:50); a mixture of autogenous and heterologous bone (70:30); heterologous bone (100\%).

Sakkas et al in 2017 (16) performed a consecutive retrospective study that was conducted on all patients who were treated at the military outpatient clinic of the Department of Oral and Plastic Maxillofacial Surgery at the military hospital in Ulm (Germany) in the years of 2009 until 2011 with autologous bone transplantation prior to secondary implant insertion. Intraoral donor sites (Crista zygomatico-alveolaris, ramus mandible, symphysis mandible, and anterior sinus wall) and extraoral donor site (iliac crest) were used. A total of 279 patients underwent routinely computer tomography scans followed by virtual implant planning after a healing period of 3-5 months.

Their review demonstrates the predictability of autologous bone material in alveolar ridge reconstructions prior to implant insertion, independent from donor and recipient site including even autologous bone chips for sinus elevation. Due to the low harvesting morbidity of autologous bone grafts, the clinical results of their study indicate that autologous bone grafts still remain the "gold standard" in alveolar ridge augmentation prior to oral implantation. On the other hand, Particle size of autogenous bone graft has big influence on bone healing.

Pallesen et al in 2002 (17) studied Influence of particle size of autogenous bone grafts on the early stages of bone regeneration. The aim of this study was to investigate the influence of particle size of autogenous bone grafts on the early stages of bone regeneration. They prepared Bicortical skull bone defects in 15 rabbits (4 in each rabbit). Two defects were filled at random with either small (0.5 to 2 $\mathrm{mm} 3$ ) or large (10 mm3) autogenous bone particles. In addition, 1 defect was left unfilled (control defect). All defects were covered bicortically by expanding polytetrafluoroethylene membranes. The animals were divided randomly into 3 groups and sacrificed after 1, 2, and 4 weeks, respectively. Histologic and stereologic evaluations were performed after the sections were blinded. They found that the total volume of newly formed bone in defects with small particles was larger and more mature compared to defects with large particles after 2 and 4 weeks.
Furthermore, the resorption of small particles was more pronounced after 4 weeks, documenting a higher level of bone substitution compared to large particles. Influence of particle size of autogenous bone grafts on the early stages of bone regeneration: a histologic and stereologic study in rabbit calvarium.

All of the 21 patients included in this study, 9 were males and 5 were females, providing greater frequency in adult males. This is in agreement with the reported distribution in the general population, where there is a significant difference in occurrence of sex, with male predominance. The greater frequency in adult males may be because they are more likely to neglect their teeth or they are more likely to sustain trauma to their teeth, compared to females, all of which may be the etiology for cyst formation (18-20).

In this study, enucleation with primary closure was the treatment of choice. It is a one-stage surgical treatment followed by periodic radiographic examinations at regular intervals to observe the progress of bone regeneration of the defect. It also allows pathologic examination of the entire specimen for histopathologic diagnosis (21).

The results of the histopathological diagnosis confirmed that 17 patients had inflammatory radicular cysts (81\%), while 3 patients had dentigerous cysts (15\%), while 1 patient had keratocyst (5\%). This in agreement with Demirkol et al in 2014 (22), they reported that radicular cysts occurred more frequently in the maxilla, and that patients in their 20s and 40s were frequent among those with radicular cysts.

In the present study, the immediate and late clinical evaluation showed complete healing of the soft tissue flaps without any post-operative complications, in both groups. This is in accordance with the findings of Kulkarni et al in 2014 (23) as they reported that patients treated using PRF after harvesting a free gingival graft showed almost complete closure of the wound at 1 week, lack of inflammation at the periphery of the healing wound and better control of bleeding at the time of the surgery.

This is in accordance with Bambal et al in 2012 (24) reported. They described the use of PRF unilaterally in bilateral periapical lesions in the same patient. The sites treated with PRF showed better healing than the other sites confirmed by a 3-D X-ray.

Also, Mazor et al in 2009 (25) stated that the use of PRF as the sole filling material during a simultaneous sinus lift and implantation procedure had stabilized a good amount of regenerated bone in the subsinus cavity up to the tip of implants in a case series through a radiological and histological evaluation after 6 months from the surgery.

Galav et al in 2016 (26) performed studies to compare the clinical efficacy of PRF with autogenous bone graft for the treatment of intra bony defects in chronic periodontitis and they concluded that Both ABG and PRF can be used predictably to reconstruct lost periodontal structures as indicated by Probing pocket depth reduction and relative attachment level gain. However, in terms of osseous defect fill, ABG yields a more definitive outcome than PRF.

Also, Kapustecki et al in 2016 (27) performed treatment for 20 patients suffered from oroantral communication by autogenous bone graft from chin area and PRF and concluded that all cases in the study group showed closure of oroantral communication and there is significant increase in both bone width and height. 


\section{CONCLUSION}

- PRF can be considered a healing biomaterial, as it features all the necessary parameters permitting bone regeneration without any adverse reaction.

- The addition of AUTOGENOUS BONE GRAFT to PRF accelerates the regenerative capacity of bone. They give a predictable clinical and radiographic evidence of bone formation and faster healing than using PRF alone.

\section{CONFLICT OF INTEREST}

The authors declare that they have no conflicts of interest.

\section{REFERENCES}

1-Choukroun J, Diss A, Simonpieri A, Girard MO, Schoeffler C, Dohan S. Platelet-Rich Fibrin (PRF): A secondgeneration platelet concentrate. Part IV: Clinical effects on tissue healing. Oral Surg Oral Med Oral Pathol Oral Radiol Endod. 2006;101:56-60.

2-Gruber R, Kandler B, Fischer MB, Watzek G. Osteogenic differentiation induced by bone morphogenetic proteins can be suppressed by platelet-released supernatant. Clin Oral Implants Res. 2006;17:188-93.

3-Bowers GM, Chadroff B, Carnevale R, Mellonig J, Corio R, Emerson J. Histologic evaluation of new attachment apparatus formation in humans. Part II. J Periodontol. 1989;60:675-82.

4-Camelo M, Nevins ML, Schenk RK, Simion M, Rasperini G. Clinical, radiographic, and histologic evaluation of human bony defects treated with Bio-Oss and Bio-Gide. Int J Periodontics Restorative Dent. 1998;18:321-31.

5-Camargo PM, Lekovic V, Weinlaender M, Nedic M, Vasilic $\mathrm{N}$, Wolinsky LE, et al. A controlled re-entry study on the effectiveness of bovine porous bone mineral used in combination with a collagen membrane of porcine origin in the treatment of intrabony defects in humans. J Clin Periodontol. 2000;27:889-96.

6-Caplanis N, Lee MB, Zimmerman GJ, Selvig KA, Wikesjo UM. Effect of allogeneic freeze-dried demineralized bone matrix on regeneration of alveolar bone and periodontal attachment in dogs. J Clin Periodontol. 1998;25:801-6.

7-Yildirim M, Spiekermann H, Biesterfeld S, Edelhoff D. Maxillary sinus augmentation using xenogenic bone substitute material Bio-Oss in combination with venous blood. A histologic and histomorphometric study in humans. Clin Oral Implants Res. 2000;11:217-29.

8-Sartori S, Silvestri M, Forni F, Icaro Cornaglia A, Tesei P, Cattaneo V. Ten-year follow-up in a maxillary sinus augmentation using anorganic bovine bone (Bio-Oss). A case report with histomorphometric evaluation. Clin Oral Implants Res. 2003;14:369-72.

9-Choukroun J, Diss A, Simonpieri A, Girard MO, Schoeffler C, Dohan SL. Platelet-rich fibrin (PRF): a secondgeneration platelet concentrate. Part V: histologic evaluations of PRF effects on bone allograft maturation in sinus lift. Oral Surg Oral Med Oral Pathol Oral Radiol Endod. 2006;101:299-303.

10-Dohan DM, Choukroun J, Diss A, Dohan SL, Dohan AJ, Mouhyi J. Platelet-rich fibrin (PRF): a second-generation platelet concentrate. Part II: platelet-related biologic features. Oral Surg Oral Med Oral Pathol Oral Radiol Endod. 2006;101:e45-50.

11-Gould D, Kelly D, Goldstone L, Gammon J. Examining the validity of pressure ulcer risk assessment scales: developing and using illustrated patient simulations to collect the data. J Clin Nurs. 2001;10:697-706.

12-Damante JH, Guerra EN, Ferreira Jr O. Spontaneous resolution of simple bone cysts. Dentomaxillofac Radiol. 2002;31:182-6.

13-Gupta V, Bains BK, Singh GP, Mathur A, Bains R. Regenerative potential of platelet rich fibrin in dentistry: Literature review. Asian J Oral Health Allied Sci. 2011;1:22-8.

14-Pommer B, Georgopoulos A, Dvorak G, Ulm C. Decontamination of autogenous bone grafts: Systematic literature review and evidence-based proposal of a protocol. Quintessence Int. 2014;45:145-50.

15-Carini F, Longoni S, Amosso E, Paleari J, Carini S, Porcaro G. Bone augmentation with TiMesh. autologous bone versus autologous bone and bone substitutes. A systematic review. Ann Stomatol. 2014;5:27-36.

16-Sakkas A, Wilde F, Heufelder M, Winter K, Schramm A. Autogenous bone grafts in oral implantology-is it still a "gold standard"? A consecutive review of 279 patients with 456 clinical procedures. Int J Implant Dent. 2017;3:23.

17-Pallesen L, Schou S, Aaboe M, Hjørting-Hansen E, Nattestad A, Melsen F. Influence of particle size of autogenous bone grafts on the early stages of bone regeneration: a histologic and stereologic study in rabbit calvarium. Int $\mathrm{J}$ Oral Maxillofac Implants. 2002;17:498-506.

18-Meningaud JP, Oprean N, Pitak-Arnnop P, Bertrand JC. Odontogenic cysts: a clinical study of 695 cases. J Oral Sci. 2006;48:59-62.

19-Tortorici S, Amodio E, Massenti M, Buzzanca M, Burruano F, Vitale F. Prevalence and distribution of odontogenic cysts in Sicily: 1986-2005. J Oral Sci. 2008;50:15-8.

20-Manor E, Kachko L, Puterman MB, Szabo G, Bodner L. Cystic lesions of the jaws - a clinicopathological study of 322 cases and review of the literature. Int J Med Sci. 2012;9:20-6.

21-Ettl T, Gosau M, Sader R, Reichert TE. Jaw cysts - filling or no filling after enucleation? A review. J Craniomaxillofac Surg. 2012;40:485-93.

22-Demirkol M, Ege B, Yanik S, Aras MH, Ay S. Clinicopathological study of jaw cysts in southeast region of Turkey. Eur J Dent. 2014;8:107-11.

23-Kulkarni MR, Thomas BS, Varghese JM, Bhat GS. Plateletrich fibrin as an adjunct to palatal wound healing after harvesting a free gingival graft: A case series. J Indian Soc Periodontol. 2014;18:399-402.

24-Bambal D, Manwar NU, Chandak M, Rudagi K. A comparative evaluation of the healing ability of bilateral periapical lesions treated with and without the use of platelet-rich fibrin. Todays FDA. 2012;24:54-7.

25-Mazor Z, Robert A, Del Corso M, Hari S, Michael D, David M. Sinus floor augmentation with simultaneous placement using choukroun`s platelet rich fibrin as a sole grafting material : a radiologic and histologic study at six months. J Periodontol. 2009;80:2056-64.

26-Galav S, Chandrashekar KT, Mishra R, Tripathi V, Agarwal $\mathrm{R}$, Galav A. Comparative evaluation of platelet-rich fibrin and autogenous bone graft for the treatment of infrabony defects in chronic periodontitis: Clinical, radiological, and surgical reentry. Indian J Dent Res. 2016;27:502-7.

27-Kapustecki M, Niedzielska I, Borgiel-Marek H, Różanowski B. Alternative method to treat oroantral communication and fistula with autogenous bone graft and platelet rich fibrin. Med Oral Patol Oral Cir Bucal. 2016;21:e608-13. 